\title{
Los Programas De Educación Para La Maternidad Como Medio De Empoderamiento Social. Un Ejemplo
}

By Matilde Fernandez y Fernandez-Arroyo

Comillas Pontificial University

Abstract- La salud de las personas se inicia en la gestación. Los programas de educación prenatal y postnatal son una poderosa herramienta para que las mujeres tomen el control de su salud y se empoderen. Los programas han demostrado su eficacia para producir cambios en los hábitos, los conocimientos, las técnicas de autocontrol, la seguridad, la autoeficacia y la vinculación con sus bebés. Este trabajo aporta un ejemplo de cómo los programas prenatales y postnatales no sólo cambian a los individuos que asisten a ellos, sino que favorecen la formación de grupos de iguales que establecen relaciones de colaboración, lo cual facilita un cambio social, que a su vez incide en la salud de los individuos de la comunidad, generando una espiral de desarrollo "individuo-grupo-comunidad". Este ejemplo puede promover investigaciones sobre los cambios sociales que facilitan los programas de educación para la salud impartidos desde la concepción de empoderar a la població.

Keywords: prenatal education, childbirth education, centering pregnancy, empowerment, achievement educational.

GJHSS-C Classification: FOR Code: 420399

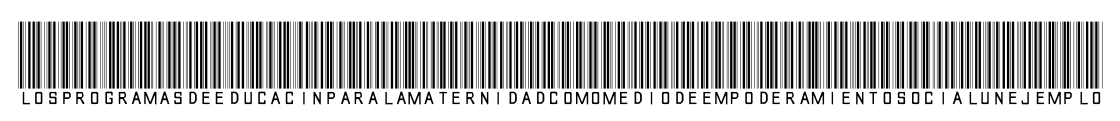

Strictly as per the compliance and regulations of:

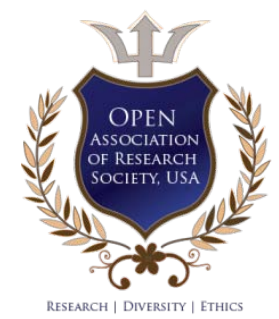

(C) 2021. Matilde Fernandez y Fernandez-Arroyo. This research/review article is distributed under the terms of the AttributionNonCommercial-NoDerivatives 4.0 International (CC BY-NC-ND 4.0). You must give appropriate credit to authors and reference this article if parts of the article are reproduced in any manner. Applicable licensing terms are at https://creativecommons.org/ licenses/by-nc-nd/4.0/. 


\title{
Los Programas De Educación Para La Maternidad Como Medio De Empoderamiento Social. Un Ejemplo
}

\author{
Matilde Fernandez y Fernandez-Arroyo
}

Abstract La salud de las personas se inicia en la gestación. Los programas de educación prenatal y postnatal son una poderosa herramienta para que las mujeres tomen el control de su salud y se empoderen. Los programas han demostrado su eficacia para producir cambios en los hábitos, los conocimientos, las técnicas de autocontrol, la seguridad, la autoeficacia y la vinculación con sus bebés. Este trabajo aporta un ejemplo de cómo los programas prenatales y postnatales no sólo cambian a los individuos que asisten a ellos, sino que favorecen la formación de grupos de iguales que establecen relaciones de colaboración, lo cual facilita un cambio social, que a su vez incide en la salud de los individuos de la comunidad, generando una espiral de desarrollo "individuo-grupo-comunidad". Este ejemplo puede promover investigaciones sobre los cambios sociales que facilitan los programas de educación para la salud impartidos desde la concepción de empoderar a la población.

Keywords: prenatal education, childbirth education, centering pregnancy, empowerment, achievement educational.

\section{INTRODUCTION}

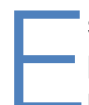

ste capítulo muestra como la educación para la salud produce cambios en la salud de los individuos que repercuten en su entorno social.

La salud de las personas comienza en la etapa prenatal, es decir, en el periodo de gestación, cuando el feto se desarrolla en el útero materno (Jacob, 2020). Durante esta etapa, desde la alimentación a las emociones de la madre, todo puede influir en la futura expresión de los genes que se configuraron en el momento de la concepción del nuevo ser humano (Higgs-Howarth, 2021). Los avances de la "epigenética" están mostrando los mecanismos que hacen posible la influencia del estado físico y psíquico materno sobre el desarrollo fetal (Casavilca-Zambrano, 2019; Hollister, 2019). A tenor de este conocimiento, la Organización Mundial de Salud (OMS), ha elaborado la Estrategia Mundial para la Salud de la Mujer, el Niño, y el Adolescente (2016-2030), que plantea mejorar la salud de la infancia desde el periodo de gestación (WHO, 2015). Desde todas las perspectivas posibles: biológica, psicológica, familiar, social, sanitaria, económica, etc., el embarazo toma una nueva dimensión de estudio y atención.

Author: Comillas Pontificial University, San Juan de Dios School of Nursing and Physical Therapy, Spain.

e-mail: mfernandezarroyo@gmail.com
Antes de hablar de embarazo, hay que reseñar la situación de las mujeres en el mundo. Según la OMS, las mujeres sufren las mayores tasas de mortalidad, morbilidad, analfabetismo y pobreza. En el informe de 2014, se estima que, cada día, mueren 800 mujeres en el mundo, por causas prevenibles relacionadas con el embarazo y el parto. Los países en vías de desarrollo computan el 99\% de esta mortalidad materna (Karlsen, \& colbs, (2011). Estas cifras visualizan la necesidad de la atención a las mujeres en la gestación y la crianza. Se puede decir que, la salud de las mujeres es una prioridad mundial que plantea retos a la asistencia obstétrica. ¿Cómo aumentar la calidad de la atención sanitaria en el embarazo y el puerperio? La evidencia muestra que cuidar a las madres es cuidar a los hijos y a las familias, la base de la estructura social. Reseñar que el concepto de calidad asistencial propugnado por la International Society for Quality in health Care (USQua) indica que la calidad comprende seis parámetros: seguridad, eficacia, centrada en el paciente, oportuna, eficiente y equitativa (USQua, 2019). Es decir, la asistencia sanitaria, para ser de calidad no puede producir ningún daño; debe ajustarse a los mejores conocimientos científicos disponibles; tiene que tener en cuenta el contexto social y las necesidades específicas de cada persona; hay que administrarla cuando se necesite sin demoras; debe llegar a todos los individuos por igual.

Dado que el embarazo es un periodo relevante en la salud futura de la persona y la situación de las mujeres en el mundo es claramente peor que la de los hombres, queda claro que hay que mejorar la calidad de la asistencia obstétrica directa y propiciar una educación prenatal y postnatal que permita que las mujeres y sus parejas tomen el control de su autocuidado en estos periodos, es decir, que se empoderen. Según Asoh (2010), el empoderamiento, es "el proceso mediante el cual las personas fortalecen sus capacidades, confianza, visión y protagonismo, en cuanto que forman parte de un grupo social para impulsar cambios positivos en las situaciones en las que viven". El concepto de empoderamiento puede analizarse desde diversas teorías. En este marco, interesa la perspectiva de género (IV Conferencia de Acción de Beijing) (2015) y la psicología comunitaria (Zimmerman, M. A., \& Rappaport, J., 1988; Israel, B. A., Checkoway, B., Schulz, A., \& Zimmerman, M., 1994; 
Peterson, N. A., \& Zimmerman, M. A., 2004) que incluyen el cambio de las personas, los grupos y la sociedad. El concepto de empoderamiento de la psicología comunitaria es una vía de desarrollo y transformación de las comunidades que, desde los componentes cognitivos, afectivos y conductuales de las personas, identifica y fomenta capacidades para la promoción del bienestar individual y grupal, que llevan al cambio social (PPD, 2013). Esta teoría de la psicología comunitaria da sustento a las intervenciones educativas de salud al identificar los elementos intrapersonales, cognitivos y conductuales del empeoramiento. Cada vez que un sanitario pregunta a su cliente: ¿qué siente?, ¿qué quiere?, está trabajando directamente sobre el componente intrapersonal de la motivación. Cada vez que pregunta ¿por qué sucede?, está actuando sobre el componente intrapersonal de locus de control. Cuando pregunta ¿qué sabe, ¿qué piensa?, ¿qué puede?, está incidiendo sobre el componente intrapersonal de la autoeficacia. La pregunta ¿qué tiene?, lleva al componente cognitivo. Las preguntas, ¿cómo lo hace?, ¿cómo puedo mejorarlo?, fomentan el uso de destrezas analíticas. Y las preguntas, ¿cómo empezamos?, y ¿qué me ayuda a seguir?, desarrollan el elemento de empoderamiento conductual que desarrolla acciones para la toma de control (Fernández, 2017).

Todas estas preguntas son relevantes en cualquier proceso de asistencia sanitaria individual. En el marco de la educación para la salud son la base del empoderamiento grupal. Su aplicación desarrolla la autoconfianza y la asertividad, lo que permite que el individuo tenga mayor autoridad en la toma de decisiones y se produzca un cambio individual que favorece la interrelación grupal. El grupo así constituido, cambia a su vez, se empodera, toma el control y produce cambios a nivel social, que a su vez repercuten en las posibilidades de mejora de los individuos. Los profesionales sanitarios, a través de los programas de educación para la salud, tienen la oportunidad de informar, educar, y crear espacios de reflexión y apoyo para que los participantes puedan desarrollar su autonomía, su autoeficacia, su control y su capacidad de participación social. Ello lleva a la creación de grupos de autoayuda que fomentan la continuidad de las mejoras obtenidas y permiten que el cambio grupal repercuta sobre la comunidad. Y el cambio comunitario a su vez influye sobre los individuos, estableciéndose una espiral de mejora que puede retroalimentarse: "individuo-grupo-comunidad" (Fernández, 2017).

Para que los profesionales sanitarios tomen conciencia de su poder como promotores del cambio social en salud, es necesario un cambio de rol profesional. El rol tradicional sitúa al sanitario en un nivel superior de capacitación del que la persona que atiende respecto a su salud, en el que la información fluye del sanitario al cliente mayoritariamente. El nuevo rol, mantiene la capacitación del profesional sanitario, pero permite que la información fluya de forma bidireccional, con lo que le da, al sanitario, la oportunidad de aprender de cada intervención y de ser el motor de un cambio de salud en la persona que recibe su atención (Fernández, 2017). Este cambio del rol profesional sanitario ya se evidencia en algunos ámbitos. Como ejemplo, podemos aportar la base de la filosofía, el trabajo y las intervenciones de las matronas australianas, para las que "matrona" (Midwife), significa: "con la mujer" (Bradfield) y han desarrollado el marco teórico "Partnership midwifery care" que promueve la salud de las mujeres a partir del desarrollo de su autonomía (Bradfield, Z., Hauck, Y., Duggan, R., \& Kelly, M., 2020; Freedman, 2006).

En Educación para la Salud, los conceptos de Calidad y Empoderamiento han revolucionado los programas educativos, haciéndolos más adaptados a las necesidades reales de los participantes. Así, los consumidores de salud pueden comprender lo que es posible y lo que no, y pueden encontrar la mejor manera de participar y tomar el control sobre su proceso de cambio en salud.

\section{il. Ejemplo de Empoderamiento}

En este capítulo se muestra cómo los programas de educación para la maternidad/paternidad pueden empoderar a la población. Se exponen los pasos a través de los cuales, el aprendizaje de los individuos, favorece un cambio grupal que lleva al cambio social.

Cuando hablamos de programas de educación para la salud, fácilmente pensamos en programas relacionados con enfermedades crónicas físicas y/o psicológicas. Programas para diabéticos, hipertensos, fumadores, obesos, bebedores, deprimidos, etc., que tienen una larga trayectoria evolutiva. Los programas de Educación Maternal o programas de Educación para la Maternidad/Paternidad son programas educativos dirigidos a la mujer y/o su pareja en la gestación y en el puerperio. Tienen por objeto contribuir a la salud materno-infantil durante los procesos de gestación, parto y postparto (Karlsen, 2011). Se planifican e imparten en el ámbito sanitario público y privado. Surgieron en Europa en los años treinta del siglo XX para facilitar el parto de las mujeres, cuando estas dejaron de parir en sus casas rodeadas de sus familiares, para ir a dar a luz solas, en los hospitales, con toda la tecnología obstétrica del momento. Son programas que nacieron con el objetivo de ayudar a las mujeres en el parto hospitalario, pero que con el tiempo han evolucionado y se han extendido por todo el mundo. La mayoría de las escuelas de educación prenatal han cambiado su paradigma. Han dejado de poner su enfoque en mejorar el parto de las mujeres, para ampliar su punto de mira. Ahora se trabaja porque la maternidad y la paternidad sean procesos conscientes desde la gestación a la crianza, pasando 
por el momento del nacimiento, el parto, que puede ser un encuentro afectivo con el hijo. Se ha incorporado toda la psicología prenatal y postnatal, además de todas las técnicas de autocontrol emocional y de dolor. Favorecen la creación de grupos de iguales que permiten la aparición de nuevas redes sociales de crianza, en un mundo en que los hijos llegan, a menudo, en individuos y parejas muy independientes. Facilitan una crianza más compartida, más saludable, e indudablemente, más feliz (Fernández, 2019).

En España, se da la particularidad, de que estos programas de educación pre y postnatal se contemplan en la cartera de servicios del Sistema Nacional de Salud con cobertura para toda la población del territorio del Estado Español desde 1983 (Fernández, 2014). Se ofertan a través de las consultas de las matronas de Atención Primaria (Instituto Nacional de Salud, 2001). Estos programas constituyen uno de los medios estatales para disminuir la morbi-mortalidad materna y neonatal tradicional. $Y$ ahora, que se ha conseguido que esas cifras sean muy bajas, se han convertido en un pilar para el cambio de rol personal que conlleva la maternidad y la paternidad y para el establecimiento de la reestructuración familiar que requiere la llegada de un hijo. El programa español consta de tres niveles de atención. El primero son sesiones educativas que se imparten antes de las 20 semanas de gestación. El segundo nivel son las sesiones que se imparten de la 28 semana de gestación al final del embarazo. El tercer nivel son las sesiones que se imparten en el puerperio. El segundo nivel es de obligado cumplimiento, por lo que es el que más se oferta. El tercer nivel está creciendo dada la demanda de las nuevas madres. En un principio, la asistencia era sobre todo de embarazadas. Ahora, cada vez son más las embarazadas que acuden con sus parejas (Fernández M, Muñoz I, Torres J., 2013).

\section{a) Los programas de Educación Prenatal promotores} del "Cambio Individual"

La eficacia de estos programas para alcanzar los objetivos educativos a nivel de los asistentes está demostrada (Fernández, 2013). El trabajo de Fernandez de 2012 evaluó los programas en toda una comunidad autónoma, la comunidad de Madrid donde recogieron 928 cuestionarios (Fernández, 2012). Los indicadores de "eficiencia", que miden el tiempo y los recursos, mostraron escasez de matronas en atención primaria en relación con la población que tenían que atender. Obtuvieron un ratio de 1 matrona cada 422 partos, cuando la OMS promueve un ratio de 1 matrona cada 160 partos. Esta escasez de profesionales hace que los grupos tengan más asistentes, una media de 20 mujeres y 10 parejas (Fernandez 2014). El número de asistentes recomendado para los grupos de educación para la salud es de un máximo de 15 personas y $/ 07$ parejas. Los indicadores de "eficiencia" mostraron que había que hacer un esfuerzo por captar a la población inmigrante, las mujeres de menor nivel cultural y las de riesgo por edad menor de 19 años y/o mayor de 40. Los indicadores de "funcionalidad", o evaluación de los propósitos y el proceso del programare mostraron muy buenos indicadores en todos los elementos de organización contemplados. Los indicadores de "efectividad", o evaluación de si se consiguen los objetivos educativos, mostraron un cambio importante de hábitos en los cuidados de la gestación, la alimentación, el ejercicio y el descanso. Así mismo, mostraron resultados estadísticamente significativos en cuanto a la adquisición de conocimientos sobre el embarazo, el parto, el postparto, la lactancia, los cuidados de recién nacido y los signos y síntomas por los que acudir a urgencias. También mostraron resultados estadísticamente significativos en cuanto al aprendizaje de ejercicios de suelo pélvico, técnicas de respiración y técnicas de relajación. El nivel de satisfacción con el programa fue muy alto, en una escala tipo Likert, donde 0 es el menor valor y 10 el mayor valor, obtuvieron una satisfacción de 8,9. Una media de 7,5 sesiones de programa y una asistencia media a 6,8 sesiones. Los indicadores de "impacto" a corto plazo, en el momento de finalizar el programa mostraron resultados estadísticamente significativos en cuanto a la sensación de control o seguridad que adquirían los madres y parejas asistentes hacia el embarazo, el parto, el postparto, la lactancia y los cuidados del bebé. Así somo la vinculación afectiva positiva hacia su hijo (Fernández M, Muñoz I, Torres J., 2014).

Todos estos datos muestran estadísticamente que los programas prenatales estudiados producen cambios a nivel de los individuos que asisten a ellos. No se han encontrado estudios que midan como estos programas producen cambios a nivel grupal y social. La aportación de este trabajo está en relación con la experiencia de estos cambios en un centro de salud del Norte de la Comunidad de Madrid, España.

\section{b) Del "Cambio Individual" al "Cambio Grupal"}

En el centro de atención primaria "Rosa de Luxemburgo" de San Sebastián de los Reyes" de la Comunidad de Madrid (España), la media de asistencia a los grupos de educación prenatal en el Segundo nivel o de las 28 semanas al final de la gestación es de una 21 embarazadas al mes y 13 parejas. Se trata, por tanto, de una población joven. Su nivel cultural y económico es medio y alto. Durante el desarrollo de los grupos presenciales y ahora "on-line", por la situación de COVID-19, se realizan actividades que promueven el trabajo activo de las parejas y el intercambio de dudas y experiencias. Con ello se favorece el conocimiento por parte de los integrantes de los grupos que, normalmente, inician una relación a través de la creación de un grupo de "guasap". Este grupo les permite seguir 
en contacto cuando termina el programa. Se forma lo que se conoce como grupo de "autoayuda". Es un grupo de iguales, en este caso mujeres y sus parejas al final de la gestación, que se conectan para compartir su final de embarazo, su parto y su inicio de crianza. En el nivel 2 o nivel del programa que se imparte al final de la gestación, el cambio grupal no suele pasar de compartir experiencias a través del "guasap". Sin embargo, estas mujeres y parejas vuelven a coincidir en el tercer nivel del programa, en el puerperio. Para facilitar la asistencia a estas sesiones de apoyo al puerperio, la lactancia y la crianza se ha dispuesto, en el centro de salud, una sesión fija todos los miércoles de 12 a 14 horas. Antes presencial y ahora online. Cuando era presencial la asistencia era muy alta. El horario muy libre. Esta sesión presencial de crianza se convierte en un espacio de salud y de encuentro de las mujeres, sus parejas y sus bebés en un momento crucial de sus vidas. En él se resuelven dudas, se comparten experiencias, se imparten los temas que las madres y los padres demandan, desde lactancia, cuidados del bebé, masaje del bebé, explicación del apego, desarrollo psicomotriz, ejercicios postparto con bebé, etc. Las mujeres pueden acudir siempre que lo deseen, no hay un número limitado de sesiones. Se trabaja la interrelación grupal de iguales y surgen amistades. Las mujeres empiezan a organizarse para quedar fuera del grupo del centro de salud y quedan en las salas de reunión de las comunidades de sus casas, en los parques, en los cafés y en los centros comerciales. La llegada de un bebé cambia la vida las personas. Los programas dan una gran oportunidad a las mujeres y sus parejas de conectar con mujeres y parejas de su comunidad, en las mismas circunstancias, para mejorar su satisfacción y sus recursos tras la llegada de su bebé.

\section{c) Del "Cambio Grupal" al "Cambio Comunitario"}

Una mujer, o una mujer y su pareja, con solo su bebé, se mueven por la comunidad con todas las dificultades que la arquitectura civil presenta en la actualidad. Estas dificultades hacen que muchas mujeres y sus parejas salgan poco de casa con su bebé, en los primeros meses de crianza. Sin embargo, un grupo de mujeres con sus cochecitos y sus bebés se sienten más fuertes, realmente se empoderan, para hacerse un espacio en parques, centros comerciales, restaurantes, y en todos los espacios sociales. Se ponen de acuerdo para solicitar a los ayuntamientos espacios de encuentro. En San Sebastián de los Reyes, el ayuntamiento, a través de la Delegación de Igualdad, ha estado organizando, durante dos años, encuentros semanales de mamas y sus bebés. También piden, a través de las páginas web de los ayuntamientos, de los centros comerciales, los restaurantes, etc., ascensores para poder acceder con los cochecitos, salas de lactancia, cambiadores de bebés en los aseos de mujeres, de discapacitados y de hombres.

\section{d) Del Cambio "Comunitario" al "Cambio de los} Individuos"

Todas estas mejoras solicitadas, repetidamente, por las nuevas madres y los nuevos padres, se van produciendo con el tiempo. Ello conlleva un cambio comunitario que mejora las posibilidades de la vida social de los nuevos padres. Todas las entidades que reciben las necesidades de los nuevos padres efectúan mejoras que repercuten directamente sobre los individuos. Aparecen señalizaciones de amplios ascensores para que puedan subir los cochecitos de los bebés, salas de lactancia, restaurantes con espacios para aparcar los cochecitos, gimnasios que imparten clases para las madres con sus bebés, etc. Este cambio comunitario ha promovido que algunos cines implanten, lo que han denominado "sesión teta", o sesiones de cine matutinas, de bajo coste, en salas adaptadas para que las madres puedan ver películas actuales mientras dan el pecho a sus bebés sin que nadie se moleste por ello.

\section{e) Del "Cambio Grupal" al "Cambio del Individio"}

No hay que olvidar el efecto que los grupos tienen sobre los individuos. Como se ha visto, los grupos dan seguridad a las personas para la toma de decisiones, lo que hace que las personas puedan ser más efectivas y hagan mejor uso de los recursos disponibles. Pero, en el caso de la maternidad y la paternidad, es de reseñar el efecto del grupo de iguales sobre las maternidades diferentes $\mathrm{y} / \mathrm{o}$ especiales. Valga, como ejemplo, el de una madre de 17 años que acude al programa con todas las inquietudes de su edad, de su cambio de vida y todos los problemas familiares, económicos y sociales que su maternidad inesperada conlleva. La experiencia es que es grupo la escucha, la apoya, la acoge, la protege y la acompaña en este momento tan decisivo de su vida. Modestamente pienso que ningún acompañamiento profesional exclusivo tiene la potencia de un acompañamiento profesional conjunto a un acompañamiento grupal de madres iguales. He visto a "niñas" perdidas por una maternidad inesperada convertirse en mujeres adultas y madres competentes capaces de retomar el control de sus vidas. Tengo mucho que agradecer a los grupos de mujeres anónimas que contestan "guasap" a cualquier hora del día o de la noche a las madres diferentes, a las inseguras y a las primerizas. Y qué decir de su labor ante las maternidades, digamos especiales. Cuando una mamá y un papá esperan un hijo sano y la vida les sorprende con un hijo con una "enfermedad rara", una malformación congénita, o cualquier otro problema de salud que va a requerir cuidados especiales de por vida. Los grupos de iguales ayudan en el duelo que supone la aceptación de un hijo diferente, permiten expresar el profundo dolor que sienten por no tener un hijo sano. Acompañan el proceso de convertirse en una 
madre normal para un niño con necesidades especiales. Esto es importantísimo para que se mantengan en el ámbito social y no se sientan relegadas al ámbito de los enfermos. El grupo facilita la aceptación de la diferencia con su contacto y su apoyo. Normaliza la maternidad especial al promover la relación con madres de niños sanos y facilita muchos recursos, ya que todas las madres buscan la forma de poder ayudar a la madre de un niño especial. Surge entre ellas una conexión que evita que muchas madres se desconecten de una maternidad afectiva, aunque tengan todas las dificultades que puede conllevar la maternidad y la paternidad de un niño enfermo.

\section{ili. COnclusion, Implicaciones y ReCOMENDACIONES}

\section{a) Conclusiones}

La Salud de las personas se inicia durante el periodo de gestación. La gestación no es solo un tiempo de desarrollo físico, sino que también es el tiempo en el que se inicia la vinculación afectiva entre la madre o la madre y la pareja con el futuro hijo. Los programas educativos prenatales han demostrado su eficacia en la promoción del cambio individual y el empoderamiento que ejercen sobre las personas. La experiencia muestra su poder para promover el cambio grupal y social para que los nuevos padres tomen el control de su salud, de la de sus hijos y promuevan la adaptación social a sus necesidades de crianza en su entorno inmediato. Los programas prenatales $y$, sobre todo los postnatales, favorecen nuevas redes sociales de crianza que van a permitir un mejor desarrollo familiar y social de los nuevos núcleos familiares.

\section{b) Implicaciones}

Conocer el poder de la educación para la salud para empoderar a la población permite diseñar estrategias de atención sanitaria y programas educativos con esta perspectiva de empoderamiento. Esto es, contemplando que el desarrollo de las personas con necesidades específicas permite la creación de grupos de iguales que pueden mantener los cambios individuales en el tiempo y facilitar cambios en la comunidad.

\section{c) Recomendaciones}

La pandemia de COVI-19 ha cambiado la forma de impartir los programas de educación para la salud de la forma presencial a la forma on-line. La experiencia muestra que la asistencia a las sesiones del final de gestación no se ha visto modificada en cuanto a número de asistentes, pero ha disminuido la interacción grupal entre los mismos. La asistencia a las sesiones de puerperio se ha visto muy disminuida en cuanto al número de asistentes on-line. Aunque se mantiene el día y la hora para atender a la población en el puerperio, son menos las madres que se conectan a las sesiones. Se recomienda retomar la forma presencial de los programas que se imparten en el puerperio por la riqueza de interacción que da la presencialidad, frente a las sesiones online. Se recomienda hacer un esfuerzo de diseño de estrategias de comunicación grupal en toda la educación para la salud on-line. Que no se pierda el objetivo de formar grupos de iguales que perpetúen los cambios individuales y favorezcan el cambio social.

\section{Agradecimientos}

A todas las madres, padres, profesionales sanitarios, agentes sociales que han permitido la realización de este trabajo. $Y$ al comité editorial de la revista que se ha interesado por su publicación.

\section{References Références Referencias}

1. Ahlers-Schmidt, C. R., Okut, H., \& Dowling, J. (2020). Impact of Prenatal Education on Breastfeeding Initiation Among Low-Income Women. American journal of health promotion: AJHP, 34(8), 919-922. https://doi.org/10.1177/089 0117120925342

2. Amanak, K., Sevil, U., \& Karacam, Z. (2019). The impact of prenatal education based on the Roy adaptation model on gestational hypertension, adaptation to pregnancy and pregnancy outcomes. JPMA. The Journal of the Pakistan Medical Association, 69(1), 11-17.

3. Asoh, D. A., \& Rivers, P. A. (2010). The empowerment and quality health value propositions of e-health. Health services management research, 23(4), 181-184. https://doi.org/10.1258/hsmr.2010. 010007

4. Bradfield, Z., Hauck, Y., Duggan, R., \& Kelly, M. (2020). Midwives' experiences of learning and teaching being 'with woman': A descriptive phenomenological study. Nurse education in practice, 43, 102699. Advance online publication. https://doi.org/10.1016/j.nepr.2020.102699

5. Casavilca-Zambrano, S., Cancino-Maldonado, K., Jaramillo-Valverde, L., \& Guio, H. (2019). Epigenética: la relación del medio ambiente con el genoma y su influencia en la salud mental. Revista de Neuro-Psiquiatria, 82(4), 266-273. https://doi. org/10.20453/rnp.v82i4.3648

6. Fernández Fernández-Arroyo, M. (2017). Los programas de educación maternal y el empoderamiento de las mujeres. MUSAS. Revista de Investigación En Mujer, Salud y Sociedad, 2(1), 3-20. https://doi.org/10.1344/musas2017.vol2.num 1.1

7. Fernández M, Muñoz I, Torres J. Estudio de validez y fiabilidad del cuestionario EDUMA2 para evaluar la calidad del segundo nivel o sesiones del tercer trimestre de gestación de los Programas de 
Educación Maternal. Matronas Hoy 2013; 2(1): 14-26.

8. Fernández M. (2012). Elaboración de una herramienta de evaluación de los programas de educación maternal que se imparten en los centros de atención primaria de la Comunidad de Madrid. Tesis. Universidad Pontificia Comillas (España). https://www.educacion.gob.es/teseo/imprimirFicher oTesis.do?idFichero=G2×2\%2FLI8uT4\%3D

9. Fernández y Fernández-Arroyo, M., Muñoz San Roque, I., \& Torres Lucas, J. (2014). Características de los programas de educación maternal de atención primaria de la Comunidad de Madrid (España) y de sus asistentes. Matronas Prof, 15(1), 20-26.

10. Fernández Y Fernández-Arroyo, M., Muñoz, I., \& Torres, J. (2014). Assessment of the pregnancy education programme with 'EDUMA2' questionnaire in Madrid (Spain). Journal of evaluation in clinical practice, 20(4), 436-444. https://doi.org/10.1111/ jep. 12170

11. Fernández, M. (2019). "Childbirth Education: Comparative Analysis". En: Chilbirth. (págs. 47-62.) London: intechopen. DOI: 10.5772/intechopen.880 21. https://www.intechopen.com/books/childbirth/ childbirth-education-comparative-analysis

12. Freeman, L. M., Adair, V., Timperley, H., \& West, S. $\mathrm{H}$. (2006). The influence of the birthplace and models of care on midwifery practice for the management of women in labour. Women and birth : journal of the Australian College of Midwives, 19(4), 97-105. https://doi.org/10.1016/j.wombi. 2006.10.001

13. Higgs-Howarth, S., Benstead, M., Booth, A. T., \& Hutchinson, D. (2021). Exploring Perinatal Indicators of Infant Social-Emotional Development: A Review of the Replicated Evidence. Clinical child and family psychology review, 24(3), 450-483. https://doi.org/ 10.1007/s10567-021-00356-2

14. Hollister, B. M., Yaremych, H. E., Goldring, M. R., \& Persky, S. (2019). Mothers' and fathers' cognitive and affective responses to epigenetics concepts. Environmental epigenetics, 5(4), dvz021. https://doi. org/10.1093/eep/dvz021

15. International Society for Quality in health Care (USQua). (2019). Resources. Dublin: USQua. https://isqua.org/resources-blog/resources.html

16. Israel, B. A., Checkoway, B., Schulz, A., \& Zimmerman, M. (1994). Health education and community empowerment: conceptualizing and measuring perceptions of individual, organizational, and community control. Health education quarterly, 21(2), 149-170. https://doi.org/10.1177/109019819 402100203

17. Jacob, C. M., Killeen, S. L., McAuliffe, F. M., Stephenson, J., Hod, M., Diaz Yamal, I., Malhotra, J., Mocanu, E., Mclntyre, H. D., Kihara, A. B., Ma, R.
C., Divakar, H., Kapur, A., Ferriani, R., Ng, E., Henry, L., Van Der Spuy, Z., Rosenwaks, Z., \& Hanson, M. A. (2020). Prevention of noncommunicable diseases by interventions in the preconception period: A FIGO position paper for action by healthcare practitioners. International journal of gynaecology and obstetrics: the official organ of the International Federation of Gynaecology and

18. Obstetrics, 151 Supp/ 1(Suppl 1), 6-15. https://doi. org/10.1002/ijgo.13331

19. Karlsen, S., Say, L., Souza, J. P., Hogue, C. J., Calles, D. L., Gülmezoglu, A. M., \& Raine, R. (2011). The relationship between maternal education and mortality among women giving birth in health care institutions: analysis of the cross sectional WHO Global Survey on Maternal and Perinatal Health. BMC public health, 11, 606. https://doi.org/10.1186/ 1471-2458-11-606

20. Partners in Population and Development (PPD) and The Partnership. (2013). Promoting Women'S Empowerment for Better Health Outcomes for Women and Children. Strategy Brief for the Inter Ministerial Conference on "South-South Cooperation in Post ICDP and MDGs", Beijing, China 22-23 October, 2013, 4.

21. Peterson, N. A., \& Zimmerman, M. A. (2004). Beyond the individual: toward a nomological network of organizational empowerment. American journal of community psychology, 34(1-2), 129-145. https://doi.org/10.1023/b:ajcp.0000040151.77047. 58

22. UN Women. (2015). Summary Report: The Beijing Declaration and Platform for Action turns 20. New York: UN Women. https://www.unwomen.org/en/ digital-library/publications/2015/02/beijing-synthesis -report

23. World Health Organization. (2015). Estrategia mundial para la salud de la mujer, el niño y el adolescente (2016-2030). Geneva: WHO, 2015. https://www.who.int/maternal child adolescent/doc uments/estrategia-mundial-mujer-nino-adolescente2016-2030.pdf

24. World Health Statistics (2014). Geneva, World Health Organization; 2014. http://apps.who.int/iris/ bitstream/handle/10665/112738/9789240692671_en g.pdf; jsessionid $=5$ D186675F133FEB30FD41BE102 BBC893? sequence $=1$

25. Yikar, S. K., \& Nazik, E. (2019). Effects of prenatal education on complaints during pregnancy and on quality of life. Patient education and counseling, 102(1), 119-125. https://doi.org/10.1016/j.pec.2018. 08.023

26. Zimmerman, M. A., \& Rappaport, J. (1988). Citizen participation, perceived control, and psychological empowerment. American journal of community psychology, 16(5), 725-750. https://doi.org/10.1007/ BF00930023 